\title{
VULNERABILIDADE NA ADOLESCÊNCIA: a experiência e expressão do adolescente
}

\author{
F lávia Barbosa de JE SU Sa, Fernanda Cristina A guiar LI M A ${ }^{b}$, Christine Baccarat de G odoy M ART IN Sc, \\ Karla Fonseca de M AT OS ${ }^{b}$, Solange Pires Salomé de SOU ZA ${ }^{d}$
}

\section{RESUM 0}

Estudo transversal, quantitativo, cujo objetivo foi identificar situações de vulner abilidade vivenciadas pelos adolescentes do ensino médio da rede pública de Cuiabá, M ato Grosso, no segundo semestre de 2009. U tilizou-se questionário fechado. Os resultados revel aram que $10,5 \%$ dos meninos e $5,8 \%$ das meninas já fizer am uso de drogas ilícitas, aos 15 anos, sendo a cocaína (28,9\%) e a maconha (15,7\%) as mais relatadas; $45,2 \%$ dos meninos e $52,4 \%$ das meninas consomem bebidas al coólicas, sendo a cerveja a mais comum. Entre os que se declararam fumantes (16,0\%), a maioria iniciou o consumo aos 15 anos. Houve relato de violência sexual, dos quais somente $33,3 \%$ dos meninos e 25,0\% das meninas procuraram ajuda. Entre os que não procuraram ajuda, o motivo relatado foi medo e vergonha. Destaca-se a necessidade de serviços específicos para prevenção e tratamento de adolescentes em situações de vulnerabilidade, tendo em vista a ocorrência e consequências destes agravos.

D escritores: Vulnerabilidade. Comportamento do adolescente. D rogas ilícitas. Alcoolismo. Violência sexual.

\section{RESUMEN}

E ste estudio transversal tuvo por objetivo identificar las situaciones de vulnerabilidad vividas por adolescentes de la escuelas públi cas de Cuiabá, $M$ ato $\mathrm{G}$ rosso, B rasil. L os resultados revelan queel $10,5 \%$ de los varones y el $5,8 \%$ delas chicas ya han usado drogas ilícitas a los 15 años (40,0\% de los varones y $27,7 \%$ de las chicas); $45,2 \%$ de los varones y $52,4 \%$ de las chicas consume bebidas al cohólicas. E ntre los que dedararon quefuman (16,0\%), la mayoría se inició a los 15 años. H ubo relatos deviolencia sexual por parte de un $3,0 \%$ de los varones y $1,2 \%$ de las chicas, de los cuales solamente el $33,3 \%$ de los varones y $25,0 \%$ de las chicas buscaron ayuda. Teniendo en vista la ocur rencia y consecuencias de los hechos constatados, se destaca la necesidad de servicios específicos para la prevención y tratamiento de adolescentes en situaciones de vulnerabilidad.

D escriptores: Vulnerabilidad. Conducta del adolescente. D rogas ilícitas. Alcoholismo. Violencia sexual.

T ítulo: Vulnerabilidad en la adolescencia: la experiencia y expresión del adolescente.

\section{ABST RACT}

This cross-sectional quantitative study aims to identify situations of vulnerability experienced by adolescents of public secondary schools in Cuiabá, state of $M$ ato $G$ rosso, B razil, during the second semester of 2009. A closed questionnaire was used in the research. The results show that $10.5 \%$ of the boys and $5.8 \%$ of the girls had already used illegal drugs at the age of 15 cocaine (28.9\%) and marijuana (15.7\%) were the most reported; and that $45.2 \%$ of the boys and $52.4 \%$ of the girls drink alcoholic beverages, beer being the most common. Among self-declared smokers (16.0\%), the majority began smoking at the age of 15 . Sexual violencewas reported, but only $33.3 \%$ of the boys and $25.0 \%$ of the girls sought help. F ear and shame werethe reported reasons for not seeking help. T he need for specific prevention and treatment services for adolescents in vulnerable situations stands out, in view of the occur rence and the consequences of this kind of harm.

D escriptors: Vulnerability. A dol escent behavior. Street drugs. A Icoholism. Sexual violence. $T$ itle: Vulnerability during adol escence: the experience and the expression of the adolescent.

\footnotetext{
${ }^{a}$ G raduanda de Enfermagem pela U niversidade Federal de M ato Grosso (UFM T), Cuiabá, M ato G rosso, Brasil.

b M estranda pelo Programa de Pós-G raduação em Enfer magem da F aculdade de Enfer magem da UFM T, Cuiabá, M ato Grosso, Brasil.

' D outora em Saúde Pública, D ocente da F aculdade de Enfermagem da U F M T, Cuiabá, M ato Grosso, Brasil.

d D outora em Enfermagem em Saúde Pública, D ocente da Faculdade de Enfermagem da U F M T, Cuiabá, M ato G rosso, Brasil.
} 


\section{INT RODUÇÃO}

A adolescência é um período marcado por intensas mudanças, dúvidas e indecisões, principalmente em relação à sexualidade. Desta forma, 0 adolescente encontra-se mais vulnerável à gravidez não planejada, às doenças sexualmente transmissíveis (DST), à experimentação de drogas, exposição aos acidentes em decorrência do comportamento desafiador, além de diferentes formas de violência(1)

Assim como o uso de drogas ilícitas, o álcool também constitui uma das principais causas desencadeadoras de situações de vulner abilidade na adolescência. A Organização M undial da Saúde (OM S) aponta o álcool como a substância psicoativa mais consumida no mundo e também como a droga de escolha entre crianças e adolescentes. No Brasil, o álcool também é a droga mais usada em qualquer faixa etária e o seu consumo entre adolescentes vem aumentando, principalmente entre os mais jovens de 12 a 15 anos de idade(2).

0 abuso sexual é outra situação a que 0 adolescente é vulnerável, sendo considerado pela OM S como um dos maiores problemas de saúde pública. E studos realizados em diferentes partes do mundo sugerem que $36 \%$ das meninas e $29 \%$ dos meninos sofrem abuso sexual. Entretanto, sua real prevalência ainda é desconhecida, visto que muitas crianças não revelam o abuso, somente conseguindo falar sobre ele na idade adulta(3).

F rente aos prejuízos como contaminação por DST, gravidez não planejada, desistência da escola e violência, além de outras vulnerabilidades, se faz necessário conhecer estas situações vivenciadas pelos adolescentes, o que contribui para o planejamento e implantação de serviços específicos de prevenção e atendimento.

Neste sentido, o presente estudo teve como objetivo identificar o consumo de drogas, álcool, tabaco e situações de violência sexual, vivenciadas pelos adolescentes do primeiro ano do ensino médio da rede pública de ensino de Cuiabá, $M$ ato Grosso.

\section{MET ODOLOGIA}

Trata-se de um estudo de corte transversal, com análise quantitativa. Foram selecionadas para a amostra cinco escolas estaduais de Cuiabá, $\mathrm{M}$ ato $\mathrm{Grosso}$, dentre as quais priorizou-se as tur- mas do primeiro ano do ensino médio (22 turmas) A amostra foi selecionada juntamente com a Secretaria de Estado de E ducação (SEDUC) de M ato G rosso, de forma aleatória. A população de estudo foi composta por 499 adolescentes (alunos das cinco escolas selecionadas), cujos critérios de inclusão foram: ser alunos do primeiro ano do ensino médio da rede pública e estar na faixa etária de 11 a 19 anos, período considerado pela Organização M undial da Saúde como adolescência.

Para a coleta dos dados foi utilizado um questionário fechado e auto-aplicável, composto por 15 questões, aplicado à população de estudo pela própria pesquisadora, auxiliada por uma equipe de voIuntários, no período de julho a dezembro de 2009. As categorias de análise foram: perfil sócio-econômico-familiar (com quem reside, se considera amado pelos pais) e vulnerabilidades (uso de drogas ilícitas e bebidas al coólicas, tabagismo e violência sexual).

Os dados foram processados el etronicamente através do programa E pi-Info, versão 3.5.1, e analisados por meio de frequência absoluta e relativa. Foram realizadas algumas análises bivariadas, em que se considerou 0 valor de $p<0,05$.

Os adolescentes participaram da pesquisa de forma voluntária, após assinarem o T ermo de Consentimento Livre e Esclarecido, sendo-Ihes assegurado 0 anonimato absoluto das informações, assim como a privacidade.

O estudo foi aprovado pelo Comitê de Ética em Pesquisa do Hospital U niversitário Julio M üller (CEP-HUJM), conforme protocolo número 613/ CEP-HUJM / 09, e autorizado pel a Secretaria de Estado de E ducação (SEDU C), bem como pelas Diretorias das respectivas escolas.

\section{RESULTADOS E DISCUSSÃO}

D os 499 questionários anal isados, 56,0\% dos adolescentes moravam com os pais, $30,2 \%$ com a mãe e apenas $6,2 \%$ com parentes. 0 fato de a maior parte dos adolescentes morarem com os pais é destacado por alguns autores como um fator positivo, visto que não residir com a família aumenta a chance de consumo de bebidas alcoólicas, tabaco e outras drogas ilícitas ${ }^{(4)}$. D estaca-se, neste sentido, que a relação dos pais com os filhos constitui-se elemento essencial, tanto pela possibilidade de diálogo quanto pela responsabilidade cobrada pela família(5). 
A maioria dos adolescentes $(62,4 \%$ dos meninos e $72,4 \%$ das meninas) tem certeza de serem amados pelos pais, enquanto $21,7 \%$ dos meninos e $14,2 \%$ das meninas apenas acham que sim, 7,9\% dos meninos e $6,5 \%$ das meninas deixaram em branco, $5,3 \%$ dos meninos e 3,9\% das meninas não sabiam, $2,1 \%$ dos meninos e $1,3 \%$ das meninas acham que não, $0,5 \%$ dos meninos e $0,6 \%$ das meninas têm certeza de não serem amados pelos pais e 0,5\% dos meninos e $1.0 \%$ das meninas não responderam $(p=$ $0,22)$.

T er certeza de serem amados pelos pais constitui-se fator considerado essencial para a redução da vulnerabilidade, pois para estudiosos, a separa- ção dos pais, rejeição pelos mesmos, problemas de relacionamento, conflitos familiares, uso de álcool ou drogas por pais e irmãos, ausência de normas e regras claras (limites), falta de apoio, de diálogo e de orientação, relações afetivas precárias e falta de monitoramento constante dos filhos por parte dos pais contribui diretamente para que 0 adolescente se envolva ou não com drogas ilícitas, bebidas alcoólicas e tabagismo(1,6).

Poucos adolescentes $(10,5 \%$ dos meninos e $5,8 \%$ das meninas) já fizeram uso de droga ilícita (T abela 1), na idade de 15 anos, com diferença estatisticamente significativa entre os sexos ( $p=$ 0,0028).

T abela 1 - Distribuição dos adolescentes segundo sexo e uso de drogas ilícitas. Cuiabá, M T, 2010.

\begin{tabular}{|c|c|c|c|c|c|c|}
\hline \multirow{3}{*}{ U so de drogas ilícitas } & \multicolumn{4}{|c|}{ Sexo } & & \\
\hline & \multicolumn{2}{|c|}{ M asculino } & \multicolumn{2}{|c|}{ Feminino } & \multicolumn{2}{|c|}{ T otal } \\
\hline & $n$ & $\%$ & $n$ & $\%$ & $\mathbf{n}$ & $\%$ \\
\hline \multicolumn{7}{|l|}{ Já fez uso } \\
\hline Sim & 20 & 10,5 & 18 & 5,8 & 38 & 7,6 \\
\hline Não & 153 & 80,5 & 279 & 90,2 & 432 & 86,5 \\
\hline E m branco & 17 & 9,0 & 10 & 3,2 & 27 & 5,4 \\
\hline Respostas nulas & - & - & 2 & 0,6 & 2 & 0,4 \\
\hline Total & 190 & 100,0 & 309 & 100,0 & 499 & 100,0 \\
\hline \multicolumn{7}{|l|}{ I dade em que fez uso } \\
\hline 14 anos & 1 & 5,0 & 1 & 5,5 & 2 & 5,2 \\
\hline 15 anos & 8 & 40,0 & 5 & 27,7 & 13 & 34,2 \\
\hline 16 anos & 6 & 30,0 & 9 & 50,0 & 15 & 39,4 \\
\hline 17 anos & 4 & 20,0 & 2 & 11,1 & 6 & 15,7 \\
\hline 18 anos & 1 & 5,0 & 1 & 5,5 & 2 & 5,2 \\
\hline Total & 20 & 100,0 & 18 & 100,0 & 38 & 100,0 \\
\hline \multicolumn{7}{|l|}{ Frequência de uso } \\
\hline Semanal & 5 & 25,0 & 1 & 5,5 & 6 & 15,7 \\
\hline E ventual mente & 12 & 60,0 & 6 & 33,3 & 18 & 47,3 \\
\hline Em branco & 3 & 15,0 & 10 & 55,5 & 13 & 34,2 \\
\hline Respostas nulas & - & - & 1 & 5,5 & 1 & 2,6 \\
\hline T otal & 20 & 100,0 & 18 & 100,0 & 38 & 100,0 \\
\hline \multicolumn{7}{|l|}{ Tempo de consumo } \\
\hline$M$ enos de 6 meses & 8 & 44,4 & 8 & 47,1 & 16 & 42,1 \\
\hline 6 meses a 1 ano & 2 & 11,0 & - & & 2 & 5,2 \\
\hline 1 a 2 anos & - & - & 1 & 5,9 & 1 & 2,6 \\
\hline 3 a 4 anos & 1 & 5,5 & - & - & 1 & 2,6 \\
\hline 5 anos e mais & 1 & 5,5 & - & & 1 & 2,6 \\
\hline E m branco & 5 & 27,7 & 8 & 47,1 & 13 & 34,2 \\
\hline Respostas nulas & 3 & 16,6 & 1 & 5,9 & 4 & 10,5 \\
\hline Total & 20 & 100,0 & 18 & 100,0 & 38 & 100,0 \\
\hline
\end{tabular}


Entre as drogas utilizadas, 28,9\% já fizeram uso de cocaína, bem como mais de uma droga, e $15,7 \%$ utilizaram maconha. A maioria dos meninos $(60,0 \%)$ e $33,3 \%$ das meninas faziam uso de droga eventualmente ( $p=0,0162)$. G rande parte dos adolescentes ( $44,4 \%$ dos meninos e $47,1 \%$ das meninas) fazia uso de drogas ilícitas há menos de 6 meses $(p=0,3373)$.

0 relato de uso de drogas entre os adolescentes pesquisados é apontado como um evento associado à falta de lazer, às condições de vida dos jovens, ao envolvimento familiar, à ausência dos pais, à violência doméstica, aos amigos que usam droga e à baixa percepção de apoio dos pais ${ }^{(7)}$. N este sentido, é preciso desenvolver programas voltados para o lazer desses adolescentes como atividades recreativas extracurriculares que mantenham 0 aluno um período maior na escola, construção de quadras esportivas na comunidade e educação em saúde sobre drogas e suas consequências, desenvolvendo a cultura do não consumo.

A idade em que os adolescentes da presente casuística iniciaram o uso de drogas ilícitas coincide com outro estudo, no qual se observou o uso de drogas pela primeira vez aos 15 anos ${ }^{(7)}$. Contudo, há um estudo que encontrou que o início de uso de drogas entre os adolescentes foi de 10 a 12 anos, evidenciando a precocidade da experimentação das drogas ${ }^{(8)}$. A escola, juntamente com o serviço de saúde, deve desenvolver ambientes de discussão entre pais e alunos sobre o tema, para que através dessa ação os pais aumentem o acompanhamento e diálogo com os filhos.

0 uso da cocaína e da maconha como as drogas mais utilizadas pelos adolescentes pesquisados é concordante com o estudo de revisão bibliográfica a partir das bases de dados no intervalo de 1996 a 2006, em que a maconha também foi apontada como droga de escolha dos adolescentes ${ }^{(9)}$. A escolha da droga, muitas vezes, está relacionada aos aspectos socioeconômicos, pois grande parte dos adolescentes não trabalha e não tem renda própria, o que permite consumir apenas drogas de baixo custo como a maconha.

Quanto à frequência de uso de drogas ilícitas na presente pesquisa, foi encontrado o uso eventual e por um período curto (inferior a 6 meses). Ressalta-se que, apesar de não encontrarmos estudos que pudessem fazer comparações nesse sentido, é necessário destacar que mesmo sendo um período curto é relevante para a dependência, pois essa não é decorrente do tempo e sim do simples consumo. Ressalta-se, ainda, a repercussão do uso de drogas na saúde, acarretando em acidentes de trânsito, comportamentos de risco como transmissão de DST, agressões, depressões clínicas e gravidez não planejada(10).

A T abela 2 mostra que a maioria dos adolescentes ( $45,2 \%$ dos meninos e $52,4 \%$ das meninas) fazia uso de bebidas alcoólicas ( $p=0,2589)$, cujo início se deu aos 15 anos $(p=0,0320)$, sendo a cerveja a mais comum.

E $m$ relação à frequência do consumo de bebidas alcoólicas, $50,0 \%$ dos meninos e $67,9 \%$ das meninas a consumiam eventual mente $(p=0,0073)$, há menos de 6 meses (17,4\% dos meninos e 19,1\% das meninas) ( $p=0,0061$ ).

0 uso de álcool entre os adolescentes estudados, com maior frequência entre as meninas e em idades precoces, concorda com estudo sobre 0 perfil sociodemográfico, comportamento e consequências do consumo de álcool, com estudantes de quinta série de ensino fundamental a terceira série de ensino médio de escolas públicas e privadas de Paulínia, São Paulo(2). É importante ressaltar que, nas últimas décadas, observa-se uma maior exposição das adolescentes em relação ao uso de álcool, através da mídia e da liberdade não vigiada, o que chama a atenção de alguns estudiosos, pois há algumas peculiaridades em relação às mulheres, resultando em dependência química mesmo em menores quantidades e com maiores consequências, quando comparado ao sexo masculino(11).

D estaca-se, entre as bebidas utilizadas pelos adolescentes na presente casuística, a facilidade com que a cerveja é adquirida em estabel ecimentos comerciais, festas, casas noturnas, postos de gasolina e quiosques perto da escola. Vale ressaltar que nem sempre 0 estabelecimento pede identificação para menores de 18 anos, apesar de a Lei Federal no 8.069/90, art. 81, do E statuto da Criança e do A dolescente determinar obrigatório(12). Para al guns autores, diversos fatores influenciam o consumo do álcool na adolescência, tais como: contexto familiar e social, expectativas e crenças, preço acessível, disponibilidade comercial, facilidade de acesso, além do acesso dentro de casa, o que pode resultar em dependência na idade adulta ${ }^{(2,13)}$. A presente pesquisa identificou o consumo de álcool aos 15 anos, dado semelhante foi encontrado em outra pesquisa na qual a idade da primeira experimen- 
T abela 2 - Distribuição dos adolescentes segundo sexo e uso de bebidas al coólicas. Cuiabá, M T, 2010.

\begin{tabular}{|c|c|c|c|c|c|c|}
\hline \multirow{3}{*}{ U so de bebidas alcoólicas } & \multicolumn{4}{|c|}{ Sexo } & & \\
\hline & \multicolumn{2}{|c|}{ Masculino } & \multicolumn{2}{|c|}{ Feminino } & \multicolumn{2}{|c|}{ T otal } \\
\hline & $\mathbf{n}$ & $\%$ & $\mathbf{n}$ & $\%$ & $\mathbf{n}$ & $\%$ \\
\hline \multicolumn{7}{|l|}{ Já fez uso } \\
\hline Sim & 86 & 45,2 & 162 & 52,4 & 248 & 49,7 \\
\hline N ão & 68 & 35,7 & 98 & 31,7 & 166 & 33,2 \\
\hline Em branco & 35 & 18,4 & 46 & 14,9 & 81 & 16,2 \\
\hline Respostas nulas & 1 & 0,5 & 3 & 0,9 & 4 & 0,8 \\
\hline T otal & 190 & 100,0 & 309 & 100,0 & 499 & 100,0 \\
\hline \multicolumn{7}{|l|}{ I dade em que fez uso } \\
\hline 11 anos & 1 & 1,2 & - & & 1 & 0,4 \\
\hline 13 anos & - & & 1 & 0,6 & 1 & 0,4 \\
\hline 14 anos & 8 & 9,3 & 18 & 11,1 & 26 & 10,4 \\
\hline 15 anos & 35 & 40,6 & 87 & 53,7 & 122 & 49,1 \\
\hline 16 anos & 23 & 26,7 & 39 & 24,0 & 62 & 25,0 \\
\hline 17 anos & 12 & 13,9 & 11 & 6,7 & 23 & 9,2 \\
\hline 18 anos & 2 & 2,3 & 4 & 2,4 & 6 & 2,4 \\
\hline 19 anos & 4 & 4,6 & - & - & 4 & 1,6 \\
\hline Respostas nulas & 1 & 1,1 & 2 & 1,2 & 3 & 1,2 \\
\hline T otal & 86 & 100,0 & 162 & 100,0 & 248 & 100,0 \\
\hline \multicolumn{7}{|l|}{ Frequência de uso } \\
\hline Diária & 4 & 4,6 & 4 & 2,4 & 8 & 3,2 \\
\hline Semanal & 23 & 26,7 & 17 & 19,7 & 40 & 16,1 \\
\hline Quinzenal & 4 & 4,6 & 8 & 5,0 & 12 & 4,8 \\
\hline$M$ ensal & 2 & 2,3 & 10 & 6,1 & 12 & 4,8 \\
\hline E ventual mente & 43 & 50,0 & 110 & 67,9 & 153 & 61,6 \\
\hline E m branco & 3 & 3,4 & 11 & 6,7 & 14 & 5,6 \\
\hline Respostas nulas & 7 & 8,1 & 2 & 1,2 & 9 & 3,6 \\
\hline T otal & 86 & 100,0 & 162 & 100,0 & 248 & 100,0 \\
\hline \multicolumn{7}{|l|}{ T empo de consumo } \\
\hline M enos de 6 meses & 15 & 17,4 & 31 & 19,1 & 46 & 18,5 \\
\hline 6 meses a 1 ano & 10 & 11,6 & 14 & 8,6 & 24 & 9,6 \\
\hline 1 a 2 anos & 9 & 10,4 & 31 & 19,1 & 40 & 16,1 \\
\hline 3 a 4 anos & 9 & 10,4 & 13 & 8,0 & 22 & 8,8 \\
\hline 5 anos e mais & 7 & 8,1 & 1 & 0,6 & 8 & 3,2 \\
\hline Em branco & 22 & 25,5 & 63 & 38,8 & 85 & 34,2 \\
\hline Respostas nulas & 14 & 16,2 & 9 & 5,5 & 23 & 9,2 \\
\hline T otal & 86 & 100,0 & 162 & 100,0 & 248 & 100,0 \\
\hline
\end{tabular}

tação do álcool está entre 15 a 20 anos(4). Outro estudo encontrou ainda idades mais precoces de 11 a 13 anos, consumo este que aumenta gradativamente com o tempo ${ }^{(11)}$. É necessário que os órgãos responsáveis pela fiscalização efetivem-na em estabelecimentos comerciais, evitando assim o consumo entre os adolescentes, além de campanhas de prevenção nas escolas e junto às famílias.
Quanto à frequência de uso de bebidas alcoólicas na presente investigação, outro estudo avaliou o consumo de álcool entre estudantes do ensino médio de uma cidade do interior do estado de São Paulo e encontrou que o nível de consumo dos adolescentes dos três anos de ensino médio foi equivalente, sugerindo que o início do uso de álcool acontece no ensino fundamental, concluin- 
do que o período de consumo é de no mínimo um ano ${ }^{(14)}$.

A T abela 3 mostra que a maioria dos adolescentes ( $58,4 \%$ dos meninos e $67,6 \%$ das meninas) não fumava $(p=0,0800)$, sendo que entre os fumantes $40,6 \%$ dos meninos $53,7 \%$ das meninas haviam iniciado o uso de tabaco aos 15 anos e $26,7 \%$ dos meninos e $24,0 \%$ das meninas aos 16 anos ( $p=$ 0,5322 ). Dos adolescentes que faziam uso de tabaco, $36,3 \%$ dos meninos e $37,0 \%$ das meninas o faziam de forma eventual, enquanto $27,2 \%$ dos me- ninos e $15,2 \%$ das meninas faziam uso diário ( $p=$ 0,4570). Em relação ao tempo de tabagismo, 33,3\% dos meninos e $42,2 \%$ das meninas fumavam há menos de 6 meses $(p=0,5646)$.

Quanto ao uso de tabaco pelos adolescentes, chama a atenção a problemática da indústria do tabaco que desenvolve propagandas voltadas para satisfazer determinados anseios, enfatizando autoconfiança e independência para que possam aderir ao seu consumo(15). Sabe-se que al gumas situações contribuem para o hábito de fumar entre os ado-

T abela 3 - D istribuição dos adolescentes segundo sexo e tabagismo. Cuiabá, M T, 2010.

\begin{tabular}{|c|c|c|c|c|c|c|}
\hline \multirow{3}{*}{ U so de tabaco } & \multicolumn{4}{|c|}{ Sexo } & & \\
\hline & \multicolumn{2}{|c|}{ M asculino } & \multicolumn{2}{|c|}{ Feminino } & \multicolumn{2}{|c|}{ T otal } \\
\hline & $n$ & $\%$ & $n$ & $\%$ & $n$ & $\%$ \\
\hline \multicolumn{7}{|l|}{ Já fumou/ fuma } \\
\hline Sim & 33 & 17,3 & 47 & 15,2 & 80 & 16,0 \\
\hline N ão & 111 & 58,4 & 209 & 67,6 & 320 & 64,1 \\
\hline E m branco & 45 & 23,6 & 51 & 16,5 & 96 & 19,2 \\
\hline Respostas nulas & 1 & 0,5 & 2 & 0,6 & 3 & 0,6 \\
\hline T otal & 190 & 100,0 & 309 & 100,0 & 499 & 100,0 \\
\hline \multicolumn{7}{|c|}{ I dade em que começou } \\
\hline 14 anos & 2 & 6,0 & 6 & 12,7 & 8 & 10,0 \\
\hline 15 anos & 14 & 42,4 & 22 & 46,8 & 36 & 45,0 \\
\hline 16 anos & 10 & 30,3 & 14 & 29,7 & 24 & 30,0 \\
\hline 17 anos & 5 & 15,1 & 5 & 10,6 & 10 & 12,5 \\
\hline 18 anos & 1 & 3,0 & - & - & 1 & 1,2 \\
\hline 19 anos & 1 & 3,0 & - & - & 1 & 1,2 \\
\hline T otal & 33 & 100,0 & 47 & 100,0 & 80 & 100,0 \\
\hline \multicolumn{7}{|l|}{ Frequência de uso } \\
\hline Diária & 9 & 27,2 & 7 & 15,2 & 16 & 20,0 \\
\hline Semanal & 4 & 12,1 & 5 & 10,8 & 9 & 11,2 \\
\hline Quinzenal & - & & 1 & 2,1 & 1 & 1,2 \\
\hline M ensal & 3 & 9,0 & 2 & 4,3 & 5 & 6,2 \\
\hline E ventual mente & 12 & 36,3 & 17 & 37,0 & 29 & 36,2 \\
\hline Em branco & 5 & 15,1 & 14 & 30,4 & 19 & 23,7 \\
\hline Respostas nulas & - & & - & & 1 & 1,2 \\
\hline T otal & 33 & 100,0 & 47 & 100,0 & 80 & 100,0 \\
\hline \multicolumn{7}{|l|}{ T empo de consumo } \\
\hline$M$ enos de 6 meses & 11 & 33,3 & 19 & 42,2 & 30 & 37,5 \\
\hline 6 meses a 1 ano & 3 & 9,0 & 2 & 4,4 & 5 & 6,2 \\
\hline 1 a 2 anos & 6 & 18,0 & 8 & 17,7 & 14 & 17,5 \\
\hline 3 a 4 anos & - & & 2 & 4,4 & 2 & 2,5 \\
\hline 5 anos e mais & 1 & 3,0 & - & & 1 & 1,2 \\
\hline Em branco & 11 & 33,3 & 14 & 31,1 & 25 & 31,2 \\
\hline Respostas nulas & 1 & 3,0 & - & & 3 & 3,7 \\
\hline T otal & 33 & 100,0 & 80 & 100,0 & 80 & 100,0 \\
\hline
\end{tabular}


lescentes, como o costume de fumar entre irmãos e amigos, familiar fumante, exposição à fumaça do tabaco fora de casa e objeto com logotipo da marca de cigarro(16).

A pesar da eventualidade no consumo do tabaco verificada no presente estudo, pesquisa realizada em Portugal(17) mostrou que os adolescentes fumam com regularidade. Alguns autores apontam que a nicotina é o componente responsável pela dependência ao fumo, sendo caracterizada pelo uso progressivo e abusivo de tabaco(18). É interessante que a escola juntamente com a família, a comunidade e os profissionais de saúde desenvolvam ações de prevenção ao tabagismo e contribuam com medidas para interrupção do hábito de fumar.
H ouve relato de violência sexual em 3,0\% dos meninos e $1,2 \%$ das meninas (T abela 4 ). D os que sofreram 0 abuso, 33,3\% dos meninos e $25,0 \%$ das meninas procurou ajuda, sendo que $16,6 \%$ dos meninos e $75 \%$ das meninas não contou a ninguém. E ntre os que procuraram ajuda, recorreram à mãe, ao tio e padrasto. Entre os que não procuraram, 0 motivo relatado foi por medo e vergonha dos pais e sociedade. Entre as mudanças relatadas após 0 abuso, a desconfiança em relação aos homens por parte das meninas e as mudanças de comportamento foram as mais frequentes.

0 relato de violência sexual em ambos os sexos e 0 fato de não procurarem ajuda por medo ou vergonha é apontado por estudiosos como fe-

T abela 4 - Distribuição dos adolescentes segundo sexo e se já sofreu violência sexual. Cuiabá, M T, 2010.

\begin{tabular}{|c|c|c|c|c|c|c|}
\hline \multirow{3}{*}{ Sofreu violência sexual } & \multicolumn{4}{|c|}{ Sexo } & & \\
\hline & \multicolumn{2}{|c|}{ M asculino } & \multicolumn{2}{|c|}{ Feminino } & \multicolumn{2}{|c|}{ T otal } \\
\hline & $\mathbf{n}$ & $\%$ & $\mathbf{n}$ & $\%$ & $n$ & $\%$ \\
\hline U ma vez & 3 & 1,5 & 4 & 1,2 & 7 & 1,4 \\
\hline Várias vezes & 3 & 1,5 & - & - & 3 & 0,6 \\
\hline Não & 133 & 70,0 & 243 & 78,6 & 376 & 75,3 \\
\hline Não lembro & 5 & 2,6 & 3 & 1,0 & 8 & 1,6 \\
\hline Em branco & 45 & 23,6 & 57 & 18,4 & 102 & 20,4 \\
\hline Respostas nulas & 1 & 0,5 & 2 & 0,6 & 3 & 0,6 \\
\hline T otal & 190 & 100,0 & 309 & 100,0 & 499 & 100,0 \\
\hline
\end{tabular}

Nota: $p=0,0456$

nômeno característico da violência sexual, pois é praticada na sua maioria por pessoas ligadas diretamente às vítimas, sobre as quais exercem alguma forma de poder e dependência ${ }^{(3)}$. A vítima, portanto, sente-se desprotegida e ameaçada por aquele de quem depende física e emocionalmente. Por imaginar que realmente não será ouvida, ou mesmo acreditada, além de envergonhada pelo que passa, a criança/ adolescente fica impossibilitada de denunciar. Apesar de na presente pesquisa as meninas terem relatado o evento em menos proporção em relação aos meninos, estudos mostram $^{(19,20)}$ o sexo feminino mais vulnerável a este tipo de abuso, o que nos faz refletir sobre a possibilidade das meninas pesquisadas não terem tido coragem de relatar a violência. N este sentido, a família e a escola precisam estar atentas às mudanças de comportamento do adolescente e buscar meios de ajuda junto aos órgãos responsáveis.

\section{CONCLUSÕES}

F rente aos resultados, é possível apontar para a necessidade de políticas e estratégias voltadas para o diálogo, em que se possam compartilhar dúvidas e curiosidades, alertando os jovens quanto às diversas situações de risco nessa fase. N esse sentido, movimentos intersetoriais podem trazer bons resultados, como a atuação conjunta da Saúde, E ducação, A ntropologia, Serviço Social, entre outras áreas, unificando e efetivando programas que incluam os adolescentes na prioridade da prevenção. D estaca-se a escola e a família como duas instituições importantes na adesão dos adolescentes a esses programas.

A o identificar as situações de vulnerabilidade vivenciadas pelos adolescentes do ensino médio da rede pública de ensino de Cuiabá, M ato Grosso, o presente estudo contribui para a reflexão dos pro- 
fissionais que lidam com este grupo etário no sentido de visualizar caminhos que reduzam estes eventos nesta fase tão importante do desenvolvimento humano.

D estaca-se a necessidade de estudos que aprofundem o tema a fim de orientar prog ramas específicos de prevenção e tratamento.

0 estudo apresenta limitação por se tratar de um estudo de corte transversal, faz-se necessário destacar que a realidade constatada pode se modificar na medida em que intervenções e políticas de atenção forem implantadas.

\section{REFERÊNCIAS}

1 Beserra EP, P inheiro PN C, Alves M DS, Barroso M G T. A dolescência e vulne4rabilidade às doenças sexualmente transmissíveis: uma pesquisa documental.J Bras D oenças Sex T ransm. 2008;20(1):32-5.

2 Vieira DL, Ribeiro M , Romano M , Laranjeira RR. Álcool e adolescentes: estudo para implementar políticas municipais. Rev Saúde Pública. 2007;41(3):396403.

3 Pfeiffer L, Salvagni E P. Visão atual do abuso sexual na infância e adolescência. J Pediatr. 2005;81(5):197204.

4 Souza DPO, Areco KN, Filho DXS. Álcool e alcoolismo entre adolescentes da rede estadual de ensino de Cuiabá, M ato G rosso. Rev Saúde Pública. 2005;39 (4):585-92

5 Jinez M L, Souza JJ RM , Pillon SC. U so de drogas e fatores de risco entre estudantes de ensino médio. Rev Latino-Am E nfermagem. 2009;17(2):246-52.

6 Pratta E M M , Santos M A. U so de drogas na família e avaliação do relacionamento com os pais segundo adolescentes do ensino médio. P sico (Porto A legre). 2009; 40(1):32-41.

7 Caval cante M BPT, Alves M DS, Barroso M G T. A dolescência, álcool e drogas: uma revisão na perspectiva da promoção da saúde. E sc A nna N ery. 2008;12(3): 555-9.

8 G alduróz JCF, N oto AR, Fonseca A M , Carlini EA.V levantamento nacional sobre o consumo de drogas psicotrópicas entre estudantes do ensino fundamental e médio da rede pública de ensino nas 27 capitais brasileiras. São Paulo: Centro Brasileiro de Informações sobre D rogas Psicotrópicas; 2004.

9 W agner M F, Oliveira M S. H abilidades sociais e abuso de drogas em adolescentes. Psicol Clin. 2007;19(2): 101-16.

10 M inistério da Saúde (BR), Secretaria de A tenção à Saúde. A política do M inistério da Saúde para atenção integral a usuários de álcool e outras drogas. 2a ed. Brasília (DF); 2004.

11 Strauch E S, Pinheiro RT, Silva RA, H orta BL. U so de álcool por adolescentes: estudo de base populacional. Rev Saúde Pública. 2009;43(4):647-55.

12 M inistério da Justiça (BR). Lei no 8.069, de 13 de julho de 1990: dispõe sobre o E statuto da Criança e do A dolescente e dá outras providências [ I nternet] . Brasília (DF); 1990 [ citado 2010 ago 18] . Disponível em: http:// www.planalto.gov.br/ ccivil_03/leis/ I8069.htm

13 M aia SA. Verificação dos efeitos de atividade preventiva no padrão de uso de álcool em uma população estudantil de Diadema, SP [ dissertação]. São Paulo: Coordenadoria de Controle de Doenças; 2009.

$14 \mathrm{M}$ artins RA, Cruz LAPN, T eixeira OS, M anzato AJ. Padrão de consumo deálcool entre estudantes do ensino médio de uma cidade do Interior do estado de São Paulo. SM AD, Rev Eletrônica Saúde M ental Alcool Drog [I nternet]. 2008 [ citado 2010 ago 18];4(1). D isponível em: http:/ / www.revistas4usp. sibi.usp.br/ sciel o.php?pid=S1806-69762008000 $100005 \&$ script $=$ sci_arttext.

15 M inistério da Saúde (BR ), Instituto N acional de Câncer. Vigescola: vigilância de tabagismo em escolares: dados e fatos de 12 capitais brasileiras. Brasília (DF); 2004.

$16 \mathrm{H}$ allal ALC, G otlieb SLD, A Imeida L M , Casado L. Prevalência e fatores associados, ao tabagismo em escolares da Região Sul do Brasil. Rev Saúde Pública. 2009:43(5):779-88.

$17 \mathrm{~F}$ raga S, Ramos E, Barros H. U so de tabaco por estudantes adolescentes portugueses e fatores associados. Rev Saúde Pública. 2006;40(4):620-6.

18 H ortense FT P, Carmagnani M IS, B rêtas ACP. 0 significado do tabagismo no contexto do câncer de laringe. Rev Bras Enferm. 2008;61(1):24-30. 
19 I noue SRV, Ristum M . Violência sexual: caracterização e análise de casos revel ados na escola. E stud Psicol. 2008;25(1):11-21.
20 Souza D P O, F ilho DX S. U so recente deálcool, tabaco e outras drogas entre estudantes adol escentes trabalhadores enão trabalhadores. Rev Bras E pidemiol. 2007;10(2):276-87.
Endereço da autora / Dirección del autor / Author's address:

Christine Baccar at de G odoy M artins Rua Fortal eza, 70, Jardim Paulista 78065-350, Cuiabá, M T E-mail: leocris2001@terra.com.br
Recebido em: 21/ 10/ 2010

A provado em: 25/ 05/ 2011 